\title{
CTV, COMPLEX TRANSIENT AND VOLTAGE STABILITY: A NEW METHOD FOR COMPUTING DYNAMIC ATC
}

\author{
M. Eidiani, ${ }^{*}$ M. Oloomi Buygi, ${ }^{* *}$ and S. Ahmadi ${ }^{* * *}$
}

\begin{abstract}
This paper presents a fast and accurate dynamic method for ATC calculations considering transient stability analysis (TSA) and voltage stability analysis (VSA) termination criteria (CTV method). This method can be used for contingency screening and ranking. Estimation of the determinant of Jacobian matrix is used for assessment of voltage stability. This method is compared with the following methods: energy difference between SEP (stable equilibrium point) and UEP (unstable equilibrium point), $t_{s}$ index of Chiang, and continuation power flow. The TSA method uses Athay's PEBS (potential energy boundary surface) and a new method named POMP. The POMP method follows the Point Of Maximum Potential energy on post-fault system trajectory. This point is approximated by Taylor's expansion of second order. Because this method does not use any convergent algorithm, it is more reliable than all existing methods that use UEP. The goals of this method are assessment of ATC considering VSA and TSA and assessment of dangerous contingencies. The ideas are demonstrated on 2, 3, 7 (Cigre), 10, 30 (IEEE) and 145 (Iowa State) bus networks.
\end{abstract}

\section{Key Words}

ATC, VSA, TSA, energy function

\section{Introduction}

Order 889 mandated each control area to compute ATC and post them on a communication system called open access same-time information system (OASIS) [1]. ATC computing methods can be divided into static and dynamic methods. Static methods can be further divided into three categories: OPF (linear and nonlinear optimization), sensitivity analysis [2], and continuation power flow (CPF) $[3,4]$. In OPF, for each transaction, generations and loads are increased until allowable transmission power between

* Electrical Engineering Department, Azad University of Bojnourd, Bojnourd, Iran; e-mail: www.eidiani.com, info@eidiani.com

** Power System Department, Electrical and Robotic Faculty, Shahrood University of Technology, Iran, m oloomi@yahoo.com

*** Faculty of Engineering, Mashad Institute of Technology, Iran, ahmadi@mashhadmit.com

(paper no. 203-3597) two areas reaches maximum. In $\mathrm{CPF}$, for each transaction, generations and loads are increased until one line reaches its MVA limit or other static terminated conditions.

Termination criteria in static methods are: transmission line flow constraints (thermal and static stability), diverging DC load flow, diverging AC load flow (including voltage collapse), and voltage limit on each bus $(0.95<V i<1.05)$.

Simplicity, transparency, flexibility, and rapidity (velocity or celerity) are the advantages of static methods. Disadvantages and defects of these methods are optimism, inaccuracy, and not considering all constraints. The dynamic method is a transient stability constrained by maximum allowable transfer (MAT). This method consists of screening a large number of contingencies and scrutinizing the dangerous ones.

TSA constrained by MAT [5] is one of the best dynamic methods. The existence of stable equilibrium in a postdisturbance system is only a necessary condition of system stability [6]. It is also important to ensure that the system can safely make the transition from the pre- to postdisturbance operation point. Several methods for dynamic ATC evaluation are those based on second-kick [7], SMIB, dot product $[8,9]$, and bifurcation theory [10].

Termination criteria in dynamic methods are transient instability, voltage instability (dynamic), and static termination conditions. Robustness and accuracy are the advantages of dynamic methods. Disadvantages and defects are: the "potentially harmful (dangerous)" contingencies in base case (first stable operating point) and ATC case (when system reaches terminated criterion) are not necessarily the same; voltage stability and transient stability are not considered simultaneously; and response is slow.

Stable operating points in base case and ATC case are different. Therefore, we use contingency ranking in stressed stable operating point.

In this work, we improve ATC with TSA and VSA termination criteria. Estimation of the determinant of Jacobian matrix is used for computing VSA index. This method is compared with energy difference between SEP and UEP [11], $t_{s}$ index of Chiang [12], and CPF [3, 4]. TSA is a very important termination criterion in computing ATC. PEBS [13], BCU [14], and second kick [7] are the 
most important methods in TSA with energy method. We use POMP for TSA termination criterion and demonstrate that this method has a very good performance.

In this paper, energy method uses voltage stability constraint as upper limit of transient stability, and computed transient stability limit by energy method is used as initial condition of simulation method. VSA methods are presented in Section 2, TSA methods in Section 3, and ATC computing methods are applied to one small system in Section 4. In Section 5, system equations and structure preserving energy functions [15] are derived. The main algorithm is presented in Section 6. In Section 7, ATC is determined for 2, 3, 7 (Cigre), 10, 30 (IEEE), and 145 (Iowa State) bus networks.

\section{VSA Methods}

Continuation power flow [3, 4], angular distance between the SEP and closest UEP in a Euclidean sense [16], energy difference between the SEP and closest UEP using an energy function [11], test function $\left(t_{s}\right)$ [12], and estimations of the determinant of Jacobian matrix are the most important methods for VSA. The means of computing the performance index proposed in [12] is easier than other proposed methods, which require computation of eigenvalues, eigenvectores, singular values, energy functions, or condition numbers [12]. It should be pointed out that the index proposed in [12] does not require computing unstable load flow solutions (UEP). This method [12] has the following deficiencies: determination of $t_{s}$ is time consuming, and the solution is not accurate.

In this section we propose a new approach for VSA.

\subsection{Estimation of the Jacobian Determinant}

The Jacobian matrix can be divided into four submatrixes $A, B, C$, and $D$. It is easy to show that:

$$
J=\left[\begin{array}{ll}
A & B \\
C & D
\end{array}\right], \operatorname{det}(J)=\operatorname{det}(A) \cdot \operatorname{det}\left(D-C A^{-1} B\right)
$$

Define:

$\operatorname{diag}(A)=\left[\begin{array}{llll}a_{11} & a_{22} & \ldots & a_{n n}\end{array}\right], a_{i i}$ are diagonal elements of $A$. and $\operatorname{prod}(\operatorname{diag}(A))=a_{11} \cdot a_{22} \cdots a_{n n}$

$$
\frac{1}{\operatorname{diag}(A)}=\left[\begin{array}{llll}
\frac{1}{a_{11}} & \frac{1}{a_{22}} & \ldots & \frac{1}{a_{n n}}
\end{array}\right]
$$

and

$$
\operatorname{diag}(\operatorname{diag}(A))=\left[\begin{array}{ccc}
a_{11} & 0 & 0 \\
0 & a_{22} & 0 \\
0 & 0 & a_{33}
\end{array}\right]
$$

Now we show:

$$
\left|X_{i i}\right|>\left|X_{i j}\right| \forall i, j \quad \text { and } \quad X=A, B, C \text { and } D
$$

We have:

$$
\begin{aligned}
A_{i i}= & \frac{\partial P_{i}}{\partial \delta_{i}}=-\sum_{j=1, \neq i}^{n} V_{i} V_{j} Y_{i j} \sin \left(\delta_{i}-\delta_{j}-\gamma_{i j}\right) \\
A_{i j}= & \frac{\partial P_{i}}{\partial \delta_{j}}=V_{i} V_{j} Y_{i j} \sin \left(\delta_{i}-\delta_{j}-\gamma_{i j}\right) \\
D_{i i}= & \frac{\partial Q_{i}}{\partial V_{i}}=\sum_{j=1, \neq i}^{n} V_{i} Y_{i j} \sin \left(\delta_{i}-\delta_{j}-\gamma_{i j}\right) \\
& +2 V_{i} Y_{i j} \sin \left(-\gamma_{i j}\right) \\
D_{i j}= & \frac{\partial Q_{i}}{\partial V_{j}}=V_{i} Y_{i j} \sin \left(\delta_{i}-\delta_{j}-\gamma_{i j}\right)
\end{aligned}
$$

If we suppose $\left|v_{i}\right| \approx 1,\left|\delta_{i}\right| \approx 0$, and $\left|\gamma_{i j}\right| \approx 90$, then:

$$
\begin{aligned}
& A_{i i}=-\sum_{j=1, \neq i}^{n} Y_{i j}, \quad A_{i j}=Y_{i j} \Rightarrow\left|A_{i i}\right|>\left|A_{i j}\right| \\
& D_{i i}=-\sum_{j=1}^{n} Y_{i j}-Y_{i i}, \quad D_{i j}=-Y_{i j} \Rightarrow\left|D_{i i}\right|>\left|D_{i j}\right|
\end{aligned}
$$

$B_{i i}$ and $C_{i i}$ can be computed similarly. Now we can propose a new approximation for Jacobian matrix. We have:

$$
\begin{aligned}
\operatorname{Det}(J)= & \operatorname{prod}(\operatorname{diag}(A)) \cdot \operatorname{prod}(\operatorname{diag}(D) \\
& -\operatorname{diag}(C) \operatorname{diag}(1 / \operatorname{diag}(A)) \cdot \operatorname{diag}(B))
\end{aligned}
$$

This estimation of Jacobian determinant is calculated very fast and can be use for computing ATC. We define $\lambda$ as:

$$
\begin{gathered}
P_{g i}=P_{g i}^{0}(1+\lambda) \quad i \in N_{g} \text { (number of generators) and } \\
P_{d j}=P_{d j}^{0}(1+\lambda) \quad j \in N_{D} \text { (number of demands) } \\
\lambda=0 \Rightarrow P=P^{0} \text { (base power flow) and } \\
\lambda=\lambda_{c r} \Rightarrow P=P_{\max } \text { (voltage stability limit). }
\end{gathered}
$$

where $P_{g i}^{0}$ and $P_{d j}^{0}$ are generation and demand at operating point. $\lambda$ is estimated by the quadratic equation:

$$
\lambda=-a(\operatorname{det}(J))^{2}+b \quad a \text { and } b \text { are unknown }
$$

Two stable load flow solutions are needed to compute $a$ and $b$. Two different load and generation conditions (in ATC direction) are used for computing them. Now there are two equations and two unknown parameters:

$$
\lambda_{1}=-a\left(\operatorname{det}\left(J_{1}\right)\right)^{2}+b \quad \text { and } \quad \lambda_{2}=-a\left(\operatorname{det}\left(J_{2}\right)\right)^{2}+b
$$

The experimental results and most simulation results show that this approximation is not as accurate as $t_{s}$ of Chiang. Now we use a new load flow solution to increase the accuracy of new method and to calculate three unknown parameters $(a, b, c)$ : 


$$
\lambda=-a(\operatorname{det}(J))^{c}+b \quad a, b \text { and } c \text { are unknown }
$$

VSA algorithm using this approximation is summarized in the following steps:

1. Two SEPs are determined in ATC direction.

2. Test function, estimation of Jacobian determinant, is approximated using equation of order two.

3. Critical point is computed using $8 . \quad(\operatorname{det}(J)=0 \Rightarrow$ $\left.\lambda_{c r}=b\right)$.

4. Test function is approximated using three different load flow solutions (step 1 and step 3 ) by equation of order three.

5. Critical point is computed using $10 . \quad(\operatorname{det}(J)=0 \Rightarrow$ $\left.\lambda_{c r}=b\right)$.

Simplicity, accuracy, and celerity are the advantages of this algorithm. The following methods have been applied on 2-bus and 145-bus (Iowa State) test systems: (1) continuation power flow; (2) energy method (quadratic approximation) [11]; (3) energy method (exponential approximation); (4) test function of $t_{s}$ (order 4) [12]; (5) estimation of determinant; and (6) test function of $t_{s}$ (order 2) [12]. The results are depicted in Figs. 1 and 2. The new method is not only very accurate but also very fast; we use it for computing dynamic ATC considering VSA termination criteria.

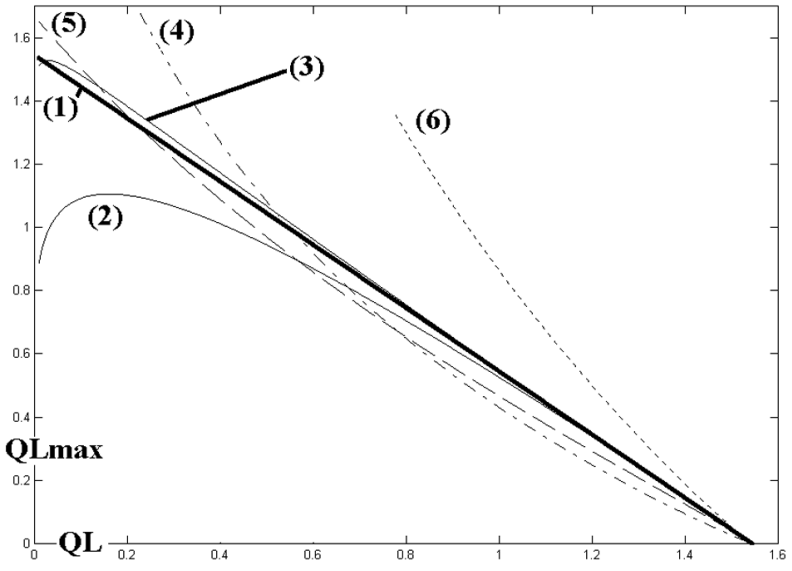

Figure 1. Computing voltage stability using six different methods for a two-bus system (QL, p.u.: reactive power demand in load bus 2) (QLmax, p.u.: maximum QL, voltage stability limit).

\section{TSA Methods}

Although Athay's PEBS method [13] and Second Kick [7] are the fastest methods for computation of critical time, they still suffer from long computation time and unreliable accuracy of solutions. We are proposing method that we believe address these deficiencies.

\subsection{Athay's PEBS Method}

A power system is modelled, in its simplest form, for the direct analysis of transient stability in the aftermath of

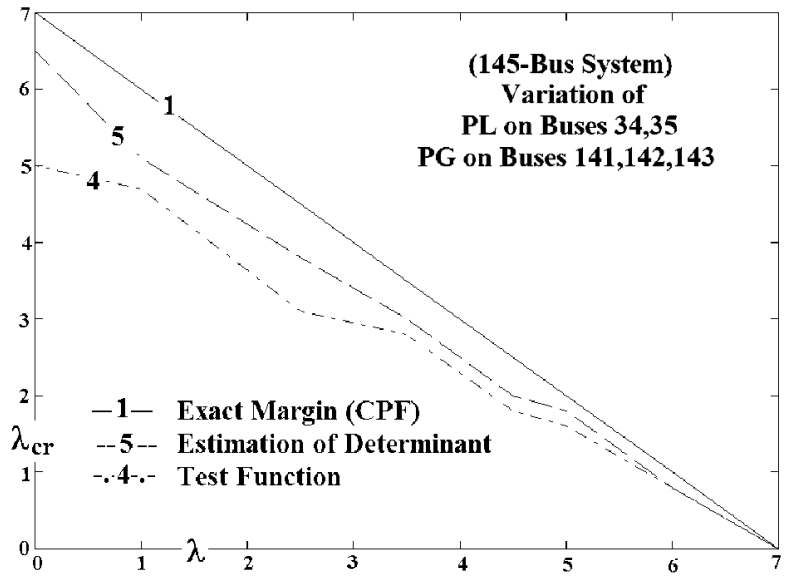

Figure 2. Computing ATC for 145-bus system (Iowa State) using methods 1,4 , and 5 .

occurrence of a fault, using the following procedure. The loads are modelled by impedance assuming constant voltages. All load and generator terminal buses are eliminated to get at the "internal node model." In an $m$ machine power system and using the centre of inertia, COI, as reference for the $i$-th machine, $i=1,2, \ldots, m$, we have:

$$
\dot{\theta}_{i}(t)=\tilde{\omega}_{i} \quad \text { and } \quad M_{i} \dot{\tilde{\omega}}_{i}(t)=f_{i}\left(\theta_{1}(t), \ldots, \theta_{m}(t)\right)
$$

where $\theta_{i}$ and $\tilde{\omega}_{i}$ are, respectively, the internal angle and the speed of generator $i$ referenced to the COI and $f_{i}\left(\theta_{1}(t), \ldots, \theta_{m}(t)\right)$ is the accelerating power of the $i$-th generator. (This section is following closely, and using the notation of, reference [17].)

To apply the direct method of Lyapunov, the transient energy function, TEF, is constructed for the system by employing the first integral of the motion of these equations to find [15]:

$V(\theta, \tilde{\omega})=\sum_{i=1}^{m} \frac{1}{2} M_{i} \tilde{\omega}_{i}^{2}-\sum_{i=1}^{m} \int_{\theta_{i}^{s}}^{\theta_{i}} f_{i}(\theta) d \theta=V_{K E}(\tilde{\omega})+V_{P E}(\theta)$

where $\theta=\left[\theta_{1}, \theta_{2}, \ldots, \theta_{m}\right]^{T}$ and $\tilde{\omega}=\left[\tilde{\omega}_{1}, \tilde{\omega}_{2}, \ldots, \tilde{\omega}_{m}\right]^{T}$.

Let $f(\theta)=\left[f_{1}(\theta), f_{2}(\theta), \ldots, f_{m}(\theta)\right]^{T}$. It has been shown [15] that inside PEBS $f^{T}(\theta)\left(\theta-\theta^{s}\right) \prec 0$, outside PEBS $f^{T}(\theta)\left(\theta-\theta^{s}\right) \succ 0$, and at the PEBS crossing, $f^{T}(\theta)\left(\theta-\theta^{s}\right)=0$ and $V_{P E}(\theta)$ reaches its maximum. We call the expression $f^{T}(\theta)\left(\theta-\theta^{s}\right)$ the PEBS crossing index. Based on these facts, Athay has proposed the following algorithm for the calculation of $t_{c r}$.

\subsubsection{The PEBS Algorithm}

1. Set up the system equations and compute the steady states $\theta^{\circ}$ and $\theta^{s}$ for the pre-fault and post-fault systems, respectively.

2. Integrate fault-on system equations using initial state $\theta^{\circ}$ and $\tilde{\omega}^{\circ}=0$ and coarse time steps.

3. At each step of integration in the above, calculate $f^{T}(\theta)\left(\theta-\theta^{s}\right)$. 
4. Repeat steps 2 and 3 until reaching $\theta^{F}$ such that $f^{T}\left(\theta^{F}\right)\left(\theta^{F}-\theta^{s}\right)=0$. Calculate $V_{c r}=V_{P E}\left(\theta^{F}\right)$. Integrate the fault-on system equations again and calculate $V(\theta, \tilde{\omega})$, this time using fine time steps, to reach $\theta_{c r}$ and $\tilde{\omega}_{c r}$ that satisfy $V\left(\theta_{c r}, \tilde{\omega}_{c r}\right)=V_{c r}$. The time to reach this point is $t_{c r}$.

\subsection{Method of POMP}

\subsubsection{Motivation}

In Fig. 3, the system trajectories (solid lines) and PEBS (dotted line) are shown for a three-machine system. Voltage angle of generator number 3 is used as reference. It is assumed that a fault occurs at time zero and clears at time $t_{c l}$. Therefore, the stable equilibrium point, SEP, of pre-fault and post-fault system is the same. This point is taken as the origin of the coordinates. In the present work, we assume the system to be lossless and with no exchange of energy with neighbouring areas. In Fig. 3, four system trajectories are shown.

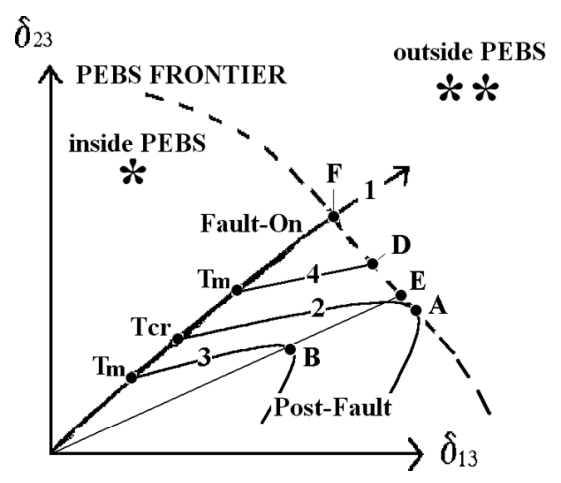

Figure 3. Sustained fault, critical trajectory, and other trajectories.

The first is the trajectory of the system under sustained fault. This trajectory starts at the pre-fault equilibrium point $\theta^{\circ}$ and $\tilde{\omega}^{\circ}=0$, which is taken here as origin $(0,0)$ and directly moves towards and crosses the PEBS. The PEBS crossing point of this trajectory (point $\mathrm{F}$ ) is assumed to be close to the saddle point controlling unstable equilibrium point.

The second is the trajectory of the critically cleared fault. Fault-on system trajectory again starts at the prefault SEP $(0,0)$, and continues until it reaches the critical time. At this time the fault is cleared, and the system moves along the post-fault trajectory until it reaches the PEBS. It meets the PEBS at point A tangentially, does not cross it, and returns towards the post-fault SEP, which is in this case the same point as the pre-fault $\operatorname{SEP}(0,0)$. At point $(\mathrm{A})$ the kinetic component of the energy function is zero and the potential energy component is maximum. This trajectory is called the critical trajectory, and the value of the energy function at the tangent point $A$ is called the critical energy, $V_{c r}$. For the system to be stable, its energy at the time fault is cleared has to be less than this critical value. Finding point A and calculating energy of the TEF, $V_{c r}$, at this point is the goal of all different approaches that use Lyapunov's direct method for analyzing system stability. Because finding the exact location of $\mathrm{A}$ is computation intensive, all different methods using TEF try to find this point approximately and calculate an approximate value for $V_{c r}$.

The third trajectory belongs to the system when the fault is cleared sooner than $t_{c r}$. It starts at the pre-fault SEP, moves along the fault-on trajectory until the fault is cleared, and then continues by moving along the postfault trajectory. As the fault clearing time is sooner than $t_{c r}$, the system is stable and its post-fault trajectory does not reach the PEBS; instead its kinetic energy decreases to a minimum, and its potential energy increases to a maximum, at point B. After this point, the post-fault trajectory returns towards the post-fault SEP.

The fourth trajectory belongs to the system when the fault is cleared later than $t_{c r}$. It starts at the pre-fault SEP, moves along the fault-on trajectory until the fault is cleared, and then continues by moving along the post-fault trajectory. As the fault clearing time is later than $t_{c r}$, the system is unstable and its post-fault trajectory reaches and crosses the PEBS at point D, where its potential energy increases to a maximum. The points A and B, where kinetic energy is minimum and potential energy is maximum, are very important. We call them points of maximum potential energy and minimum kinetic energy (POMP).

To motivate our new method and see the importance of POMPs in its structure, assume that we have a way of (approximately) calculating POMPs. Also, let us modify the third step of the PEBS algorithm to read: "At each step of integration in the above, calculate $f^{T}\left(\theta^{B}\right)\left(\theta^{B}-\theta^{s}\right)$." That is, calculate the PEBS index for the POMP B instead of the point $\theta$ on the fault-on trajectory. As the integration progresses, POMP will move towards the point A where the critical trajectory is tangent to the PEBS. Before reaching point A the PEBS crossing index $f^{T}\left(\theta^{B}\right)\left(\theta^{B}-\theta^{s}\right)$ is negative, at point $\mathrm{A}$ it is zero, and past point $\mathrm{A}$ it is positive. Therefore, monitoring this index and noting when it changes sign provides us with a way to recognize when we hit point $(\mathrm{A})$. Once we have found point $\mathrm{A}, V_{c r}$ can easily be calculated and the analysis can be carried on.

\subsubsection{POMP Determination Method}

To determine POMP by integrating the post-fault system would be very time consuming. Here we are proposing an approximate method for its determination. Consider Fig. 4. System trajectory (fault-on and post-fault) and POMP are shown for a stable system. The maximum magnitude of angles $\delta_{13}$ and $\delta_{23}$ occurs at points A1 and A2, respectively. Again, voltage angle of generator number 3 is used as reference. A1 and A2 do not occur simultaneously. $\delta_{13}$ will reach its maximum (point A1) sometime after $\delta_{23}$ has reached its maximum (point A2). Consider point C. This point, in the plane of $\delta_{13}$ and $\delta_{23}$, is defined by the set of maximum angles; $\mathrm{C}=(\mathrm{A} 1, \mathrm{~A} 2)$ and is a good approximation for POMP. 


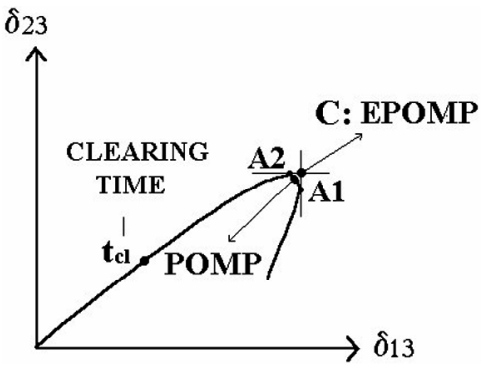

Figure 4. Fault-on and post-fault system trajectories and EPOMP.

In this method, we use $\mathrm{C}$ as an approximation to POMP and call it Estimate of POMP, or EPOMP. To find EPOMP, we expand the time variation of the angles in Taylor series and retain the first three terms. We have, using (11):

$$
\begin{aligned}
& \delta_{i m}=\delta_{i}-\delta_{m}=\theta_{i}-\theta_{m} \\
& \dot{\delta}_{i m}=\dot{\theta}_{i}-\dot{\theta}_{m}=\tilde{\omega}_{i}-\tilde{\omega}_{m}=\tilde{\omega}_{i m} \\
& \dot{\delta}_{i m}=\dot{\tilde{\omega}}_{i}-\dot{\tilde{\omega}}_{m}=\frac{1}{M_{i}} f_{i}(\theta)-\frac{1}{M_{m}} f_{m}(\theta)=F_{i m}(\theta)
\end{aligned}
$$

Expanding $\delta_{i m}(t)$ the post-fault system in Taylor series in terms of $t$ around the clearing time $t_{c l}$, retaining the first three terms, we obtain:

$$
\begin{aligned}
\delta_{i m}(t)= & \delta_{i m}\left(t_{c l}\right)+\frac{d \delta_{i m}}{d t}\left(t_{c l}\right)\left(t-t_{c l}\right) \\
& +\frac{d^{2} \delta_{i m}}{2 d t^{2}}\left(t_{c l}\right)\left(t-t_{c l}\right)^{2} \\
= & \delta_{i m}\left(t_{c l}\right)+\tilde{\omega}_{i m}\left(t_{c l}\right)\left(t-t_{c l}\right) \\
& +\frac{1}{2} F_{i m}\left(\theta\left(t_{c l}\right)\right)\left(t-t_{c l}\right)^{2} \\
= & \left(\theta_{i}\left(t_{c l}\right)-\theta_{m}\left(t_{c l}\right)\right)+\left(\tilde{\omega}_{i}\left(t_{c l}\right)-\tilde{\omega}_{m}\left(t_{c l}\right)\right)\left(t-t_{c l}\right) \\
& +\frac{1}{2} F_{i m}\left(\theta\left(t_{c l}\right)\right)\left(t-t_{c l}\right)^{2}
\end{aligned}
$$

$\theta\left(t_{c l}\right)$ and $\tilde{\omega}\left(t_{c l}\right)$ are calculated at the clearing time and are final states of fault-on system and initial states of postfault system. Differentiating (14) with respect to $t$ and equating to zero provides us with $t_{i}$, the time when angle $\delta_{i m}(t)$ reaches its maximum value:

$$
\begin{aligned}
& \dot{\delta}_{i m}(t)=\tilde{\omega}_{i m}\left(t_{c l}\right)+F_{i m}\left(\theta\left(t_{c l}\right)\right)\left(t_{i}-t_{c l}\right)=0 \\
& \text { and } \quad\left(t_{i}-t_{c l}\right)=-\frac{\tilde{\omega}_{i m}\left(t_{c l}\right)}{F_{i m}\left(\theta\left(t_{c l}\right)\right)}
\end{aligned}
$$

Note that as the system is stable, $F_{i m}\left(\theta\left(t_{c l}\right)\right)$ has to be, and is negative. Therefore, $\left(t_{i}-t_{c l}\right)$ is positive. Also note that for each $\delta_{i m}$, for each $i$, the time $t_{i}$ to reach its maximum value could be different, as the angles reach their maximums at different times. Finally, inserting (15) into (14), we obtain:

$$
\begin{aligned}
\delta_{i m}^{\mathrm{Max}}= & \delta_{i m}\left(t_{c l}\right)+\omega_{i m}\left(t_{c l}\right)\left(t_{i}-t_{c l}\right) \\
& +\frac{1}{2} F_{i m}\left(\theta\left(t_{c l}\right)\right)\left(t_{i}-t_{c l}\right)^{2} \\
= & \left(\theta_{i}\left(t_{c l}\right)-\theta_{m}\left(t_{c l}\right)\right)-\tilde{\omega}_{i m}\left(t_{c l}\right)\left(\frac{\tilde{\omega}_{i m}\left(t_{c l}\right)}{F_{i m}\left(\theta\left(t_{c l}\right)\right)}\right) \\
& +\frac{1}{2} F_{i m}\left(\theta\left(t_{c l}\right)\right)\left(\frac{\tilde{\omega}_{i m}\left(t_{c l}\right)}{F_{i m}\left(\theta\left(t_{c l}\right)\right)}\right)^{2} \\
= & \left(\theta_{i}\left(t_{c l}\right)-\theta_{m}\left(t_{c l}\right)\right)-\frac{\tilde{\omega}_{i m}\left(t_{c l}\right)^{2}}{2 F_{i m}\left(\theta\left(t_{c l}\right)\right)}
\end{aligned}
$$

The EPOMP (point $\mathrm{C}$ ) is now computed approximately by solving the following equations for $\theta_{i}, i=1, \ldots, m$ :

$$
\theta_{i}^{\mathrm{Max}}-\theta_{m}^{\mathrm{Max}}=\delta_{i m}^{\mathrm{Max}}, \quad \sum_{i=1}^{m} M_{i} \theta_{i}^{\mathrm{Max}}=0
$$

Now, we have:

$$
\mathrm{EPOMP}=\mathrm{C}=\left(\theta_{1}^{\mathrm{Max}}, \ldots, \theta_{m}^{\mathrm{Max}}\right)=\left(\theta^{\mathrm{Max}}\right)
$$

\subsubsection{POMP Algorithm}

We can state the POMP method of finding $t_{c r}$ as follows:

1. Set up the system equations and compute the steady states $\theta^{\circ}$ and $\theta^{s}$ for the pre-fault and post-fault systems, respectively.

2. Integrate fault-on system equations for a small time duration $\Delta(t)$, using initial states $\theta^{\circ}$ and $\tilde{\omega}^{\circ}=0$ and either coarse time steps or any other fast integrating method. Call values of the time and states at the end of integration $t_{b}, \theta_{b}$, and $\tilde{\omega}_{b}$.

3. Use $t_{b}$ as $t_{c l}$ in (15)-(18), to find EPOMP (point C) as an approximation of $\mathrm{B}$.

4. At each step of integration in the above, calculate $f^{T}\left(\theta^{\mathrm{Max}}\right)\left(\theta^{\mathrm{Max}}-\theta^{s}\right)$.

5. Repeat, using $t_{c l}=t_{b}$, steps 2,3 , and 4 until reaching $\theta^{*}$ such that the PEBS index becomes zero or changes sign. $\theta^{*}$ is a very accurate estimate of $\theta^{A}$ for which $f^{T}\left(\theta^{A}\right)\left(\theta^{A}-\theta^{s}\right)=0$. Calculate $V_{c r}=V_{P E}\left(\theta^{*}\right)$ as an approximation of $V_{P E}\left(\theta^{A}\right)$.

6 . Integrate the fault-on system equations and calculate $V(\theta, \tilde{\omega})$, using fine time steps, to reach $\theta_{c r}$ and $\tilde{\omega}_{c r}$ that satisfy $V\left(\theta_{c r}, \tilde{\omega}_{c r}\right)=V_{c r}$. The time to reach this point is $t_{c r}$.

This approach is more accurate than the PEBS approach. Here we use this method for computing ATC considering TSA termination criteria.

\subsection{Case Studies}

To assess the accuracy and speed of the POMP method, we apply it to two test systems to analyze stability and compute the clearing time for different faults. Single line diagrams are shown in Figs. 5 and 6. For comparison, results for the PEBS method and the nonlinear simulation, which is considered to be the exact solution, are also 


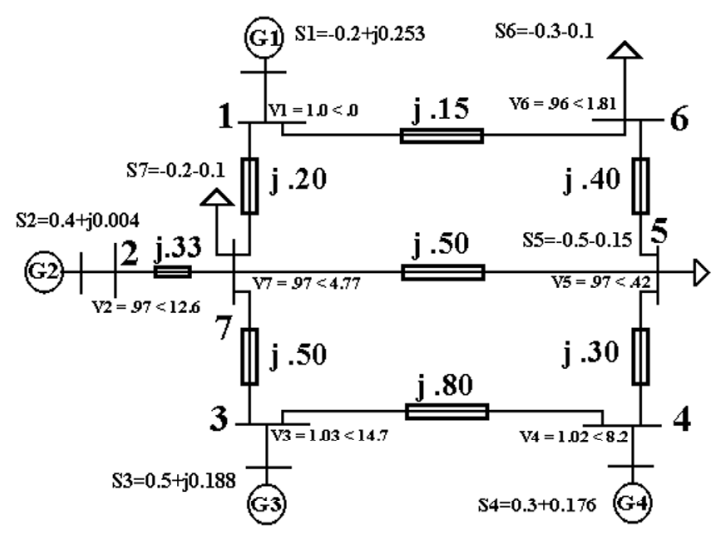

Figure 5. Single-line diagram of four machines [18].

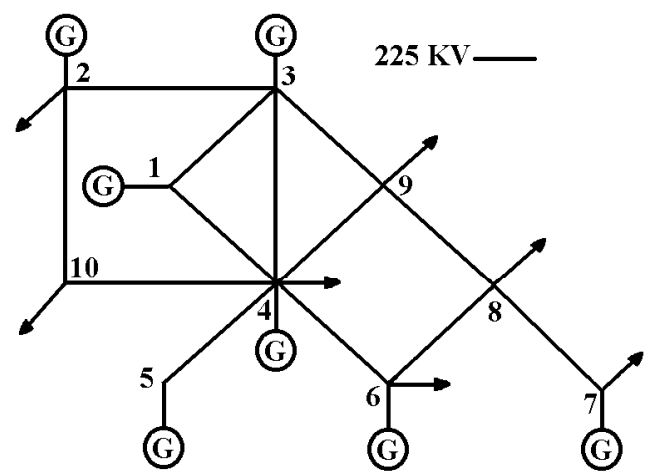

Figure 6. Single-line diagram of seven machines (Cigre).

provided. In both test systems classical model is used for the generators and COI is used as reference frame.

Table 1 shows the results for these systems. We can see that POMP is about $37 \%$ faster than PEBS method and also more accurate. In this table $\mathrm{T}$ is the calculation time

Table 1

$T_{c r}$ and $T$ (Calculation Time), by Methods (Simulation, PEBS, and POMP) for Four Machines

\begin{tabular}{|c|c|c|c|c|c|c|c|c|}
\hline \multicolumn{2}{|c|}{ Fault } & \multicolumn{2}{c|}{ Simulation } & \multicolumn{2}{c|}{ PEBS } & \multicolumn{3}{c|}{ POMP } \\
\hline Line & Percent & $\begin{array}{c}T_{c l}, \\
\text { Stable }\end{array}$ & $\begin{array}{c}T_{c l}, \\
\text { Unstable }\end{array}$ & $T_{c r}$ & $T$ & $T_{c r}$ & $T$ & $P C$ \\
\hline $3-7$ & 5 & 0.20 & 0.21 & 0.22 & 0.26 & 0.20 & 0.16 & 38 \\
\hline $3-7$ & 25 & 0.33 & 0.34 & 0.34 & 0.37 & 0.33 & 0.27 & 27 \\
\hline $3-7$ & 50 & 0.38 & 0.39 & 0.39 & 0.40 & 0.38 & 0.27 & 33 \\
\hline $3-7$ & 95 & 0.29 & 0.30 & 0.29 & 0.33 & 0.29 & 0.21 & 36 \\
\hline $4-3$ & 5 & 0.32 & 0.33 & 0.32 & 0.33 & 0.31 & 0.22 & 33 \\
\hline $4-3$ & 95 & 0.46 & 0.47 & 0.47 & 0.44 & 0.45 & 0.27 & 39 \\
\hline $5-7$ & 50 & 0.55 & 0.56 & 0.62 & 0.55 & 0.52 & 0.27 & 51 \\
\hline
\end{tabular}

$P C=\frac{T_{\mathrm{PEBS}}-T_{\mathrm{POMP}}}{T_{\mathrm{POMP}}} \times 100$
$\mathrm{T}_{c r}$ and $\mathrm{T}$ (Calculation Time), by Methods (Simulation, PEBS, and POMP) for Four Machines

\begin{tabular}{|c|c|c|c|c|c|c|}
\hline Fault & \multicolumn{2}{|c|}{ Simulation } & \multicolumn{3}{c|}{ PEBS } & \multicolumn{3}{c|}{ POMP } \\
\hline Bus & $T_{c l}$, Stable & $T_{c l}$, Unstable & $T_{c r}$ & $T$ & $T_{c r}$ & $T$ \\
\hline 1 & 0.35 & 0.36 & 0.34 & 6.6 & 0.40 & 3.7 \\
\hline 3 & 0.39 & 0.40 & 0.39 & 6.8 & 0.45 & 4.2 \\
\hline 4 & 0.49 & 0.50 & 0.44 & 8.7 & 0.50 & 4.6 \\
\hline 5 & 0.34 & 0.35 & 0.34 & 5.5 & 0.33 & 4.1 \\
\hline 6 & 0.51 & 0.52 & 0.93 & 14 & 0.59 & 6.0 \\
\hline
\end{tabular}

for PEBS and POMP methods and PC is the percentage by which POMP is faster than PEBS.

\section{A Small System}

\subsection{Two-Bus System with VSA}

Consider a system with a single transmission line, which is connected to buses 1 and 2. Bus 1 is assumed to be a slack bus with voltage magnitude fixed at 1.0 p.u. Transmission losses are neglected. The load is attached at bus 2 and is represented as a P-Q demand; that is, load is independent of bus voltage magnitude or instantaneous frequency. Power balance equations at bus 2 are:

$$
\text { and } \quad \begin{aligned}
f_{1}(\delta, v) & =P_{L}-B_{12} v \sin (\delta)=0 \\
f_{2}(\delta, v) & =Q_{L}-B_{22} v^{2}-B_{12} v \cos (\delta)=0
\end{aligned}
$$

The energy-based security measure, which indicates vulnerability of system by voltage collapse, obtained by integrating the above functions is:

$$
V(\delta, v)=-0.5 B_{22} v^{2}-B_{12} v \cos (\delta)+Q_{L} \ln (v)+P_{L} \delta
$$

It is easy to show that:

$$
\frac{\partial V}{\partial \delta}=f_{1}(\delta, v), \quad \frac{\partial V}{\partial v}=\frac{f_{2}(\delta, v)}{v}
$$

And Jacobian matrix is:

$$
J=\left[\begin{array}{ll}
\frac{\partial f_{1}}{\partial \delta} & \frac{\partial f_{1}}{\partial v} \\
\frac{\partial f_{2}}{\partial \delta} & \frac{\partial f_{2}}{\partial v}
\end{array}\right]=\left[\begin{array}{cc}
B_{12} v \cos (\delta) & B_{12} \sin (\delta) \\
B_{12} v \sin (\delta) & -2 B_{22} v-B_{12} \cos (\delta)
\end{array}\right]
$$

The following function for PEBS can be obtained by differentiating from potential energy:

$$
\operatorname{PEBS}(\delta, v)=f_{1}(\delta, v)\left(\delta-\delta^{S}\right)+\frac{f_{2}(\delta, v)}{v}\left(v-v^{S}\right)
$$

In Fig. 7 contour of potential energy, PEBS, SEP, UEP, $\operatorname{det}(J)=0$, trajectories from SEP to UEP under increasing power demand are shown for the two-bus system. As the 
potential energy is not periodic versus voltage magnitude, PEBS is not equal with local maximum potential energy in this system. Most simulation results imply that EPEBS, Estimation of PEBS, is a better approximation than PEBS. EPEBS for two-bus system is:

$$
\operatorname{EPEBS}(\delta, v)=f_{1}(\delta, v)\left(\delta-\delta^{S}\right)+\frac{f_{2}(\delta, v)}{v}\left(v-v^{S}\right) \cdot e^{-v}
$$

EPEBS is shown in Fig. 7. We use EPEBS instead of PEBS.

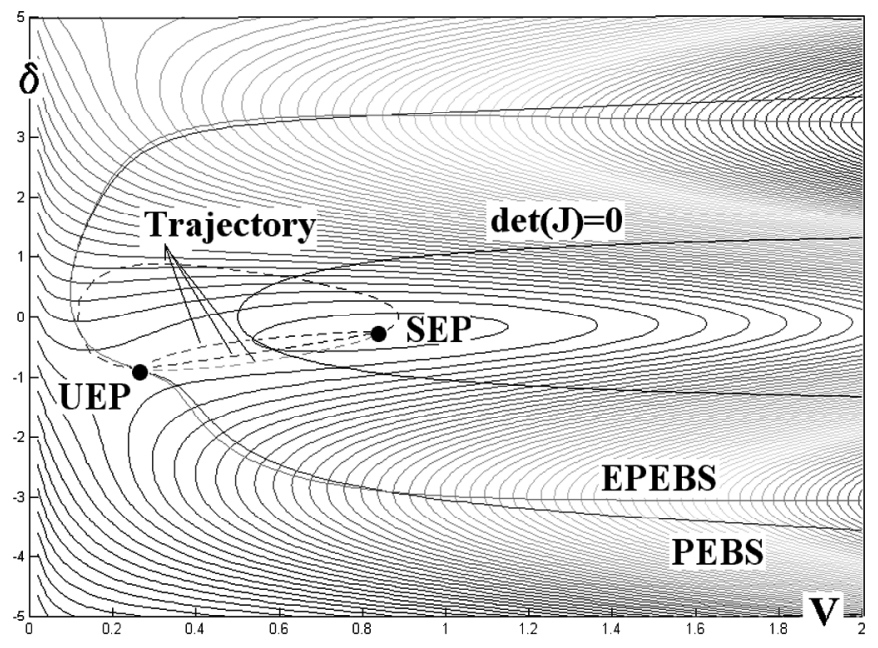

Figure 7. Equilibrium points, PEBS, EPEBS, potential energy, and $\operatorname{det}(J)=0$ in base case for two-bus system.

\section{Equations}

The assumption of a constant impedance load or ignoring of voltage magnitude may introduce an error in critical time computation. We use structure-preserving energy functions and system equations. Suppose there are $N$ number of load buses and $n$ number of generator buses. We can write the swing equations in the COI (centre of Inertia) reference frame as [15]:

$$
\begin{aligned}
& \dot{\tilde{\phi}}_{i}=\tilde{\omega}_{i} \quad i=1: n \\
& M_{i} \dot{\tilde{\omega}}_{i}=P_{m i}-\sum_{j=1}^{n+N} B_{i j} v_{i} v_{j} \sin \left(\tilde{\phi}_{i}-\tilde{\phi}_{j}\right)-\frac{M_{i}}{M_{T}} P_{C O I}
\end{aligned}
$$

The load flow equations are now written as:

$$
\begin{gathered}
P_{i}+\sum_{j=1}^{n+N} B_{i j} v_{i} v_{j} \sin \left(\tilde{\phi}_{i}-\tilde{\phi}_{j}\right)=0 \\
\text { and } Q_{i}-\sum_{j=1}^{n+N} B_{i j} v_{i} v_{j} \cos \left(\tilde{\phi}_{i}-\tilde{\phi}_{j}\right)=0, \\
i=n+1: n+N
\end{gathered}
$$

When $P$ and $Q$ in load bus are constant, the energy function is $[15]$ :

$$
\begin{gathered}
V(\tilde{\omega}, \tilde{\phi}, v)=V_{k}(\tilde{\omega})+V_{p 1}(\tilde{\phi}, v)+V_{p 2}(\tilde{\phi}) \\
\text { that: } V_{k}(\tilde{\omega})=0.5 \sum_{i=1}^{n} M_{i} \tilde{\omega}_{i}^{2} \\
V_{p 2}(\tilde{\phi})=\sum_{i=n+1}^{n+N} P_{i}\left(\tilde{\phi}_{i}-\tilde{\phi}_{i}^{S}\right) \\
V_{p 1}(\tilde{\phi}, v)=-\sum_{i=1}^{n} P_{m i}\left(\tilde{\phi}_{i}-\tilde{\phi}_{i}^{S}\right)+\sum_{i=n+1}^{n+N} Q_{i} \ln \left(\frac{v_{i}}{v_{i}^{S}}\right) \\
-0.5 \sum_{i=1}^{n+N} \sum_{j=1}^{n+N} B_{i j}\left(v_{i} v_{j} \cos \left(\tilde{\phi}_{i j}\right)\right. \\
\left.-v_{i}^{S} v_{j}^{S} \cos \left(\tilde{\phi}_{i j}^{S}\right)\right)
\end{gathered}
$$

It is well known that DAE (differential-algebraic equation) problems are very difficult to study and domain for which the DAE model may be defined as an equivalent ODE (ordinary differential equations) model [19]. The approach proposed here is to convert the DAE problem to an ODE problem. DAE models are then equivalent to the following ODE model ( $\varepsilon$ is very small):

$$
\begin{aligned}
\varepsilon \dot{\tilde{\phi}}_{i} & =P_{i}+\sum_{j=1}^{n+N} B_{i j} v_{i} v_{j} \sin \left(\tilde{\phi}_{i}-\tilde{\phi}_{j}\right) \\
\text { and } \quad \varepsilon \dot{v}_{i} & =Q_{i}-\sum_{j=1}^{n+N} B_{i j} v_{i} v_{j} \cos \left(\tilde{\phi}_{i}-\tilde{\phi}_{j}\right), \\
i & =n+1: n+N
\end{aligned}
$$

\section{Main Algorithm of CTV Method}

The experimental results and most simulation results show the efficiency of the new algorithm. This algorithm is applied on 3, 7 (Cigre), 10, 30 (IEEE), and 145 bus (Iowa State). Clearing time is assumed to be five cycles. The algorithm can be divided into three stages:

Step A: In this stage, static ATC with considering voltage stability constraint is computed using approximation of determinant of Jacobian matrix. This method is very fast, but it is very optimistic. In this stage, the system is under stress, contingencies are ranked, and the worst contingencies are determined. Computed ATC and worst contingencies are used as the initial condition of the next stage.

Step B: In this stage, dynamic ATC with considering transient stability constraint is computed using direct energy method (POMP). Computed ATC is much more accurate than stage $\mathrm{A}$. This method is quite fast, as it uses the simplified model of power system. Computed ATC is used as the initial condition of the next stage.

Step $C$ : In this stage the full detailed model of power system is used and dynamic ATC is computed accurately using simulation method. The value of ATC, which was computed in stage B, is adjusted step by step until the system reaches its stability border. 
The algorithm is shown in Fig. 8. The presented method is fast and accurate. In this algorithm, energy method uses voltage stability constraint as upper limit of transient stability, and computed transient stability limit by energy method is used as initial condition of simulation method.

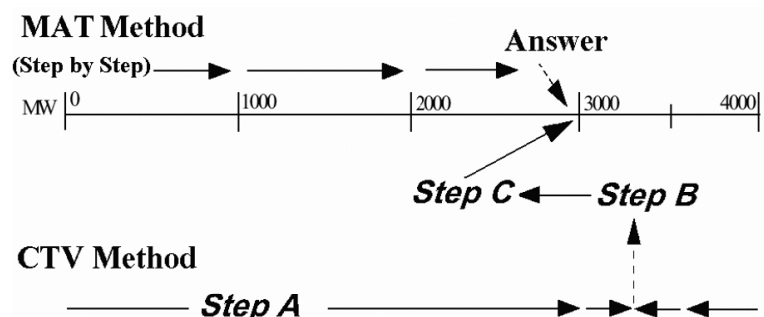

Figure 8. Main algorithm.

The new algorithm for computing ATC with considering TSA and VSA termination criteria is summarized as follows:

Step A:

1. ATC with VSA termination criteria is determined using estimation of Jacobian determinant. (Use 3 SEP and equation of order three, Section 3.2.)

2. SEP trajectory is determined for changing power using an equation of order 3 . (Use 3 SEP.)

3. The dangerous contingency is determined using SEP no. 3. This SEP is near static stability boundary. Critical voltage magnitude (low voltage) or angle (high angle) in each bus is aggravated using short circuit or load increasing. The harmful contingency may be greater than one, but it is very limited. This SEP (No. 3 ) is initial condition for 4 .
Step B:

4. Fault-on system trajectory is determined using a fast integration method (equation of order 2) until clearing time. This point is initial condition for 5 .

5 . The point of maximum potential energy is determined using POMP method.

6. EPEBS criterion is computed. If it is negative, go to 7. If it is positive, go to 8 , and if absolute PEBS is lower than maximum error, go to 9 .

7. SEP is changed backward to SEP no. 1 using step 2, and then step 5 is repeated.

8. SEP is changed forward to static stability boundary using step 2, and then step 5 is repeated.

Step $C$ :

9. In this stage the full detailed model of power system is used and dynamic ATC is computed accurately using simulation method. The value of ATC, which was computed in stage $\mathrm{B}$, is adjusted step by step until the system reaches its stability border.

Medium dynamic is not considered in this algorithm, for example, OLTCs. But because this algorithm is very fast, this issue is not important.

\section{Case Studies and Discussion}

ATC methods have been applied on five test systems: 3, 7 (Cigre), 10, 30 (IEEE), and 145 (Iowa State University) buses. Simulation results show the difference between the new CTV method, MAT [5], and accurate approaches (continuation power flow, simulation for all faults). Clearing time is assumed to be five cycles for all systems. Three methods, CTV, MAT, and accurate method, have computed dynamic ATC. Time of computation of ATC is determined for comparison of the celerity of CTV and MAT

Table 2

CTV (New Method), MAT (Old), and Accurate Method for Computing ATC

\begin{tabular}{|c|c|c|c|c|c|c|c|c|c|}
\hline \multirow{3}{*}{$\begin{array}{l}\text { Static } \\
\text { ACT } \\
\text { (p.u.) }\end{array}$} & \multicolumn{6}{|c|}{ Dynamic ATC } & \multirow{2}{*}{\multicolumn{2}{|c|}{ ATC Between }} & \multirow[t]{3}{*}{ System } \\
\hline & MAT Method & Accurate Method & \multicolumn{4}{|c|}{ CTV (New Method) } & & & \\
\hline & ATC (p.u.) & ATC (p.u.) & Speed $^{(*)}$ & $\begin{array}{c}\text { Step C } \\
\text { ATC }(\mathrm{pu}) \\
\end{array}$ & $\begin{array}{c}\text { Step B } \\
\text { ATC (pu) }\end{array}$ & $\begin{array}{c}\text { Step A } \\
\text { ATC (pu) }\end{array}$ & To $(\mathrm{PL})$ & from $\left(P_{G}\right)$ & \\
\hline 18 & 6.04 & 5.10 & 1.00 & 5.09 & 4.79 & 18 & 2 & 3 & 3 bus \\
\hline 5.0 & 1.34 & 1.42 & 1.49 & 1.41 & 1.4 & 5.0 & 5 & 2 & 7 bus \\
\hline 5.5 & 1.42 & 1.53 & 1.31 & 1.53 & 1.44 & 5.5 & 5,6 & $2,3,4$ & \\
\hline 3.1 & 0.60 & 0.82 & 2.65 & 0.82 & 0.77 & 3.1 & 8,9 & $5,6,7$ & 10 bus \\
\hline 4.2 & 0.83 & 0.92 & 5.14 & 0.91 & 0.90 & 4.2 & 3 & 2 & 30 bus \\
\hline 3.8 & 0.63 & 0.85 & 6.11 & 0.84 & 0.79 & 3.8 & 16 & 2 & \\
\hline 3.7 & 0.89 & 0.95 & 6.19 & 0.95 & 0.97 & 3.7 & 18 & 2 & \\
\hline 3.6 & 1.25 & 1.16 & 6.68 & 1.15 & 1.10 & 3.6 & 23 & 2 & \\
\hline 3.1 & 0.80 & 0.86 & 5.18 & 0.86 & 0.85 & 3.1 & 24 & 2 & \\
\hline 2.09 & 0.50 & 0.64 & 6.25 & 0.63 & 0.60 & 2.09 & 30 & 2 & \\
\hline 6.5 & 1.64 & 1.83 & 8.88 & 1.82 & 1.75 & 6.5 & $141-143$ & 34,35 & 145 bus \\
\hline
\end{tabular}

$\left({ }^{*}\right)$ Speed of CTV per MAT $=\frac{\text { Calculation time of MAT }}{\text { Calculation time of CTV }}$ 
approaches in Table 2, which shows that the CTV method is faster than the old method. As the number of buses increases, the difference between the time of new and old approaches increases. The accuracy of the new method is $22 \%$ more than that of the old one. The accurate method is only $1 \%$ more accurate than the new method. ATC considering only VSA is determined for comparing static and dynamic ATC. Static ATC is double or triple dynamic ATC. Table 2 shows that ATC dynamic is more bounded than static ATC. Similar results were also obtained for other systems. Therefore, the celerity and accuracy of the new method are very good.

\section{Conclusion}

We have proposed a new dynamic method for computing ATC. This method is able to calculate ATC for set-toset, bus-to-bus, set-to-bus, and bus-to-set and has good accuracy and celerity. The method considers TSA and VSA for computing ATC using energy method and simulation method. The proposed ATC computing method uses the most accurate methods for VSA and TSA.

\section{References}

[1] The changing structure of the electric power industry: An update, www.eia.doe.gov/cneaf/electricity/chg_stru_update/.

[2] G.C. Ejebe, J.G. Waight, M. Santos-Nieto, \& W.F. Tinny, Fast calculation of linear ATC, IEEE Trans. on Power Systems, 15(2), 2000, 1112-1116.

[3] P.Sh. Kundur, Power system stability and control (New York: McGraw-Hill, 1996).

[4] C.W. Taylor, Power system voltage stability analysis (New York: McGraw-Hill, 1994).

[5] A.L. Bettiol, L. Wehenkel, \& M. Pavella, Transient stability analysis constrained maximum allowable transfer, IEEE Trans. on Power Systems, 14(2), 1999, 654-659.

[6] I.A. Hiskens, M.A. Pai, \& P.W. Sauer, Dynamic ATC, IEEE Power Engineering Society Winter Meeting, 2000, 1629-1632.

[7] Y. Mansour, E. Vaahedi, A.Y. Chang, B.R. Corns, B.W. Garrett, K. Bemaree, T. Atahy, \& K. Cheung, B.C. hydro's on-line TSA model development analysis and post-processing, IEEE Trans. on Power Systems, 10(1), 1995, 241-253.

[8] E.D. Tuglie, M. Dicorat, M.L. Scala, \& P. Scarpellini, A static optimization approach to assess dynamic ATC, IEEE Trans. on Power Systems, 15(3), 2000, 1069-1076.

[9] X. Zhang, Y.H. Song, Q. Lu, \& S. Mei, Dynamic ATC evaluation by dynamic constrained optimization, available at http://www.ieee.org/portal/cms_docs/pes/subpages/lettersfolder/090103/PESL-00040-2003.r. 1.pdf.

[10] A. Kumar, S.C. Srivastava, \& S.N. Singh, ATC assessment in a competitive electricity market using a bifurcation approach, IEE Proc. Generation, Transmission and Distribution, 151 (2), 2004, 133-140.

[11] T.J. Overby, I. Dobson, \& C.L. DeMarco, Q-V curve interpretations of energy measure for voltage security, IEEE Trans. on Power Systems, $9(4), 1994,331-340$.

[12] H.D. Chiang, Toward a practical performance index for predicting voltage collapse in electric power system, IEEE Trans. on Power Systems, 10(2), 1995, 584-590.

[13] T. Athay, R. Podmore, \& S. Virmani, A practical method for direct analysis of transient stability analysis, IEEE Trans. on Power Apparatus 85 Systems, PAS-98(2), 1979, 584-588.

[14] H.D. Chiang, F.F. Wu, \& P.P. Varaiya, A BCU method for direct analyze of power system transient stability analysis, IEEE Trans. on Power System, 9(3), 1994, 1194-1208.

[15] A.A. Fouad \& V. Vittal, Power system transient stability analysis using the transient energy function method (NJ, USA: Prentice-Hall, 1992).
[16] T. Tamura, H. Mori, \& S. Iwamato, Relationship between voltage stability and multiple load flow solutions in electric power systems, IEEE Trans. on Power Apparatus and Systems, PAS-102(5), 1983, 1115-1125.

[17] P.W. Sauer \& M.A. Pai, Power system dynamics and stability (Upper Saddle River, NJ: Prentice-Hall, 1998).

[18] M. Zargar, Power system transient stability analysis by using the direct method of Lyapunov, doctoral diss., University of Missouri-Columbia, 1987.

[19] K.L. Praprost \& A. Loparto, An energy function method for determining voltage stability during a power system transient, IEEE Trans. on Circuit and System: Fundamental Theory and Applications, 41(10), 1994, 635-651.

\section{Biographies}

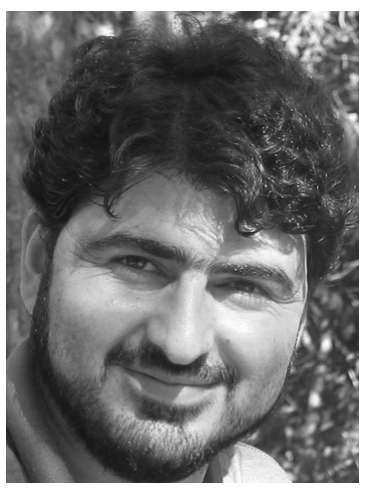

Mostafa Eidiani received his B.Sc. and M.Sc. degrees (electrical engineering) from the Ferdowsi University of Mashad in 1994 and 1996, respectively, and his Ph.D. (electrical engineering) from the Sciences and Researches Campus of Azad University. He currently teaches electrical engineering, power systems analysis, and electrical machinery at Azad University of Bojnord. He received Festival Ferdowsi Award 2nd (1995) and 4th (2003), Khwarizmi Young Festival Award (4th) in 2002, and Preferential Researcher in Azad University in Khorasan (2003). He has published over 20 papers in conference and journal. $\mathrm{He}$ is a student member of IEEE and IEE and a member of the Young Research Club and Iranian Organization for Engineering Order of Building. His research interests include power system operation and dynamics.

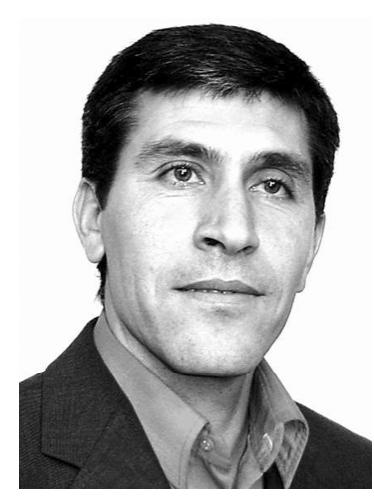

Majid Oloomi Buygi recieved his Ph.D. degree in Electrical Engineering from the Darmstadt University of Technology, Germany in 2004. He is the head of Power System Department at Shahrood University of Technology now. His main research interests include power system planning and risk management.

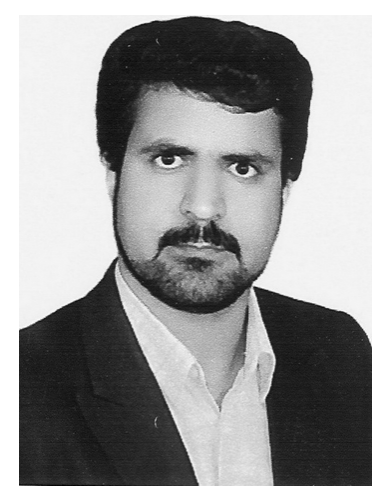

Saeed Ahmadi received his B.Sc. and M.Sc. degrees in Electrical Engineering from the Ferdowsi University of Mashad, Iran in 1993 and 1996. He is currently an educator of electrical engineering and teaches power systems analysis and electrical machinery in Mashad Institute of Technology. His research interests include power system operation and protection. 